\title{
Parents of terminally ill adolescents experienced changed parental roles and a distorted time world
}

\author{
Hayout I, Krulik T.A test of parenthood: dilemmas of parents of terminally ill adolescents. Cancer Nurs 1999 Feb;22:71-9.
}

\section{Question}

What are the processes used by parents of adolescents who are terminally ill with cancer for understanding perceptions of time and parenting?

\section{Design}

A qualitative study using methods from the phenomenological paradigm.

\section{Setting \\ Israel.}

\section{Participants}

Parents in 11 families who had an adolescent who was terminally ill.

\section{Methods \\ Data were collected over 2.5 years and included meetings with families 1-3 times each week from the time of consent until after the death of the adolescent. Meetings were held in clinics, hospi- tals, homes, and the community. Initially, general questions were used to focus on the process families experienced during long term care. Topics included family routine, sharing of household tasks and care of the sick adolescent, financial management, supports, and the adolescent's contact with friends and the edu- cational system. Data were analysed on the parental role and departure from the normative lifecycle.}

\section{Main findings}

Parents felt their role changed from parenting a healthy child to maturity to caring for a child who would decline and die (a reversal of the normal care and nurturing process). In their minds they separated the time before and after the illness. The illness was seen as a separate period of time and therefore temporary. During this period the adolescent had special status and characteristics. Concurrently, parents maintained the household routine that they had before the illness. Parents felt ambivalent desires for closeness and distance with the adolescent. They lived as part of the "healthy" society at the same time as living alongside the process of dying. When the adolescent's disease progressed, the balance between living with health and dying became more extreme.

Parents also felt unable to fulfil their normal parenting role of protecting the child and raising her to maturity. Despite the care they gave the adolescent, disease progression was inevitable.

Parents "tested" their abilities and parenting against those of other families, some of which had no ill children. Often in these comparisons, the parents felt they were good parents. These comparisons became more pronounced as the disease progressed. By making comparisons, they found their own strengths, resources, and abilities. Parents also found strength in maintaining their households and raising all of their children well. Paradoxically, the death of the adolescent did not harm the perceived parental role, but enhanced it. This enhancement served to help parents after the death of the adolescent.

\section{Conclusions}

Parents who experienced the complex process of living with an adolescent with a terminal illness felt that their parental role of raising and nurturing a child to maturity was being tested. These parents adopted positive constructions of themselves as parents and families, which assisted in defining themselves as successful parents and helped them function after the adolescent's death.

Source of funding:none stated.

For correspondence:Dr Ilana Hayout, 5 Pinsker Street, Ra'anana 43270, Israel.

\section{Commentary}

The study by Hayout and Krulik appropriately used the methods of observation and unstructured interviews for research involving such intimate and frequent contacts with parents during a difficult and emotional period. The authors did not acknowledge the therapeutic part they may have played in helping parents to examine and construct their views. 3 meetings each week between the researcher and parents during the phase of terminal care are likely to have had some effect on the outcome.

The authors did not discuss the practical application of their findings for nursing practice. However, this is an indepth study with findings of high credibility. Various implications for nurs- ing arise from the study. It is useful for nurses to understand that parents experience extreme shifts from focusing on family routines and building the future to focusing solely on their sick child.

The testing of the parent role and the feelings of failure expressed by parents reflect the grief and loss being experienced. The role of empathetic listening by nurses in these situations must not be underestimated. The intensive listening involved in a study such as this mirrors the type of work nurses engage in with patients and families. Indeed, the similarity between qualitative research skills and nursing has been noted. ${ }^{1}$

The authors found that parents of terminally ill adolescents coped with the "dying process" by constructing a positive image of themselves in the parenting role. The extent to which talking to the researchers helped parents in this process is unknown but suggests a valuable part to be played by nurses in providing a sounding board for parents to verbalise the "failure" of their child to survive.

Ann Ewens, RGN, PhD Community Health Studies Lecturer Department of Professional Education and Community Studies The University of Reading Berkshire, UK

1 Robson C. Real world research: a resource for social scientists and practitioner-researchers. Oxford: Blackwell Publisher, 1993. 